\title{
Assessment of pulmonary vascular response to exercise with Doppler-echocardiography: state of the art?
}

\author{
Alberto M. Marra ${ }^{1}$, Ekkehard Grünig ${ }^{2,3}$ \\ ${ }^{1}$ Department of Cardiovascular Imaging, IRCCS S.D.N., Naples, Italy; ${ }^{2}$ Centre for pulmonary hypertension, Thorax Clinic Thoraxklinik at \\ Heidelberg University Hospital, Heidelberg, Germany; ${ }^{3}$ Translational Lung Research Center (TLRC), the German Center for Lung Research (DZL), \\ Heidelberg, Germany \\ Correspondence to: Prof. Ekkehard Grünig, MD. Centre for Pulmonary Hypertension, Thorax Clinic at the University Hospital Heidelberg, \\ Röntgenstraße 1, Heidelberg 69126, Germany. Email: ekkehard.gruenig@med.uni-heidelberg.de. \\ Provenance: This is an invited Editorial commissioned by the Section Editor Hai-Long Dai (Department of Cardiology, Yan'an Affiliated Hospital of \\ Kunming Medical University, Kunming, China). \\ Comment on: van Riel AC, Opotowsky AR, Santos M, et al. Accuracy of Echocardiography to Estimate Pulmonary Artery Pressures With Exercise: \\ A Simultaneous Invasive-Noninvasive Comparison. Circ Cardiovasc Imaging 2017;10. pii: e005711.
}

Submitted Sep 01, 2017. Accepted for publication Sep 07, 2017.

doi: $10.21037 /$ jtd.2017.09.45

View this article at: http://dx.doi.org/10.21037/jtd.2017.09.45

There is growing evidence that the evaluation of lung circulation and right ventricular function at rest and during exercise can be helpful to detect exercise induced pulmonary hypertension (1-4), unmask an early pathological involvement of the pulmonary circulation $(5,6)$ and to stratify the prognosis $(1,7)$. Routinely, its evaluation is performed by assessment of the tricuspid regurgitation velocity (TRV), which allows an estimation of systolic pulmonary arterial pressure (sPAP) (8). The recent work by van Riel and collaborators addresses the question, whether estimation of pulmonary pressures during exercise using stress-Doppler echocardiography is accurate and allows diagnostic statements (9). The strength of this study is, that the investigators performed echocardiography and right heart catheterization simultaneously. The study showed high correlations of invasive and noninvasive assessments, both at rest and during exercise, as long as TRV signals revealed a good quality (9). At rest in $68 \%$ of patients TRV Doppler envelopes revealed a high quality and highly correlated with the invasive measurements. However, during exercise only 22 patients among 65 (34\%) presented a good quality. Lower quality TRV signals correlated poorly with invasive measurements. Thus, according to this study stress-Doppler echocardiography is very limited to assess pulmonary artery pressures in daily practice if only one third of patients can be assessed adequately. Patients with abnormal mean pulmonary artery pressures defined as increase $>3 \mathrm{mmHg} / \mathrm{L}$ mean cardiac output/minute were identified with a high sensitivity and specificity using a threshold of $1.4 \mathrm{mmHg} / 10 \mathrm{~W}$ of exercise workload. Also in this case, in patients with modest or bad quality TR profiles, it was impossible to find any relevant echocardiographic index able to predict the presence of an invasively assessed exercise-PH.

\section{Pulmonary pressures measured by echocardiography during exercise-still a long way to go?}

The work of van Riel et al. (9) sheds both lights and shadows at the assessment of the pulmonary vascular response to exercise. According to this work, the presence of a high quality TRV signal, characterised by both extension of the tricuspid signal lasting at least half of systole, as well as the presence of well-defined TRV borders, confers a reliable predictive power of resting and exercise pulmonary pressures. On the other hand, the lack of just only one from the aforementioned criteria, makes the TRV evaluation almost useless to assess pulmonary hemodynamics. However, the retrospective nature and the small sample size of the work strongly limit its results and do not allow definitive 
conclusions. Furthermore, the study cohort is quite inhomogeneous, engaging patients with several medical illnesses, both cardiovascular and respiratory as well as metabolic. For this reason, it would be of utmost importance to confirm these results in larger cohorts, and to investigate the results according to the different clinical settings.

The quality of measurements is both dependent on the investigator and the individual conditions of the patient. There is still a lack of standardization of this technique, especially the measurements during exercise are complicated and rarely performed in clinical practice (2). While at rest a remarkable rate of patients has a good quality TRV signal (44/65, 68\%), this number drastically decreases during exercise (22 patients, $34 \%$ of the whole study cohort). This is possibly also due to the method of upright cycle ergometer. Echocardiographic measurements are easier with a supine bicycle ergometer, on which patients can lie in an elevated position. However, in many clinics these supine bicycle ergometers are not available limiting this diagnostic tool further.

In conclusion, the paper of van Riel et al. (9) represents an important step forward in the awareness of possibilities and limitations of transthoracic Doppler-echocardiography for the evaluation of the pulmonary circulation. However, more research and evidences are urgently needed to test the reliability of TRV assessment in different clinical settings and to settle its standardization. Non-invasive assessment of TRV requires a supine bicycle table, thorough training and precise methodology, to achieve reproducible results.

\section{Acknowledgements}

None.

\section{Footnote}

Conflicts of Interest: The authors have no conflicts of interest to declare.

Cite this article as: Marra AM, Grünig E. Assessment of pulmonary vascular response to exercise with Dopplerechocardiography: state of the art? J Thorac Dis 2017;9(10):3607-3608. doi:10.21037/jtd.2017.09.45

\section{References}

1. Grünig E, Tiede H, Enyimayew EO, et al. Assessment and prognostic relevance of right ventricular contractile reserve in patients with severe pulmonary hypertension. Circulation 2013;128:2005-15.

2. Grünig E, Weissmann S, Ehlken N, et al. Stress Doppler echocardiography in relatives of patients with idiopathic and familial pulmonary arterial hypertension: results of a multicenter European analysis of pulmonary artery pressure response to exercise and hypoxia. Circulation 2009;119:1747-57.

3. Borlaug BA, Nishimura RA, Sorajja P, et al. Exercise hemodynamics enhance diagnosis of early heart failure with preserved ejection fraction. Circ Heart Fail 2010;3:588-95.

4. Herve P, Lau EM, Sitbon O, et al. Criteria for diagnosis of exercise pulmonary hypertension. Eur Respir J 2015;46:728-37.

5. Chia EM, Lau EM, Xuan W, et al. Exercise testing can unmask right ventricular dysfunction in systemic sclerosis patients with normal resting pulmonary artery pressure. Int J Cardiol 2016;204:179-86.

6. Nagel C, Henn P, Ehlken N, et al. Stress Doppler echocardiography for early detection of systemic sclerosisassociated pulmonary arterial hypertension. Arthritis Res Ther 2015;17:165.

7. Rieth A, Richter MJ, Gall H, et al. Hemodynamic phenotyping based on exercise catheterization predicts outcome in patients with heart failure and reduced ejection fraction. J Heart Lung Transplant 2017;36:880-9.

8. Yock PG, Popp RL. Noninvasive estimation of right ventricular systolic pressure by Doppler ultrasound in patients with tricuspid regurgitation. Circulation 1984;70:657-62.

9. van Riel AC, Opotowsky AR, Santos M, et al. Accuracy of Echocardiography to Estimate Pulmonary Artery Pressures With Exercise: A Simultaneous Invasive-Noninvasive Comparison. Circ Cardiovasc Imaging 2017;10. pii: e005711. 